\title{
AN OPEN-CHANNEL FLOW MEETING A BARRIER AND FORMING ONE OR TWO JETS
}

\author{
L. H. WIRYANTO ${ }^{1}$ and E. O. TUCK ${ }^{1}$
}

(Received 5 January 1998; revised 1 November 1998)

\begin{abstract}
A steady two-dimensional free-surface flow in a channel of finite depth is considered. The channel ends abruptly with a barrier in the form of a vertical wall of finite height. Hence the stream, which is uniform far upstream, is forced to go upward and then falls under the effect of gravity. A configuration is examined where the rising stream splits into two jets, one falling backward and the other forward over the wall, in a fountain-like manner. The backward-going jet is assumed to be removed without disturbing the incident stream. This problem is solved numerically by an integral-equation method. Solutions are obtained for various values of a parameter measuring the fraction of the total incoming flux that goes into the forward jet. The limit where this fraction is one is also examined, the water then all passing over the wall, with a $120^{\circ}$ corner stagnation point on the upper free surface.
\end{abstract}

\section{Introduction}

When a uniform stream with velocity $U$ generated far upstream meets a barrier in the form of an inclined wall of height $W$ in a channel of finite depth $H$, the stream is thrown upward against gravity $g$, and there are several different possible local flow configurations. Which of these flows occurs depends on non-dimensional parameters such as the relative height $w=W / H$ and angle $\beta$ of the wall, and the upstream Froude number

$$
F=\frac{U}{\sqrt{g H}} .
$$

A stream with a backward-going jet is typical of solutions occurring when the wall is effectively infinitely high. A free surface which is the upper boundary of the jet is then formed after the stream reaches its maximum (stagnation) height on the wall.

\footnotetext{
'Applied Mathematics Department, The University of Adelaide, Adelaide, 5005, Australia.

(C) Australian Mathematical Society 2000, Serial-fee code 0334-2700/00
} 
Hence the separation point of the free surface from the wall represents detachment with a discontinuous change in flow direction, and is a stagnation point of the flow. Solutions of this problem can be seen in Dias and Christodoulides [1], Dias and Tuck [3] and Wiryanto and Tuck [9] for a vertical wall.

Solutions with a backward-going jet but smooth non-stagnant detachment from the top of the wall without change in direction are another possible configuration, and can occur if we reduce the height of the wall to a value which is a little lower than the stagnation level. This type of solution can be obtained when the wall is itself inclined backward, that is, with an angle $\beta>\pi / 2$ measured from the positive horizontal axis. It does not appear to be possible for this flow to occur for a vertical wall. Wiryanto and Tuck [10] were able to accurately compute solutions with $\beta=3 \pi / 4$, up to close to a configuration with a stagnation point on the upper free surface.

For all of these backward-going jets, any theoretical treatment as a well-defined irrotational flow necessarily assumes that the jet is somehow absorbed or removed before it has a chance to contaminate the incident stream by falling onto its surface. This is not difficult to build into the mathematics, and is an idealisation of the real situation which retains some validity for relatively thin jets.

If the wall height is reduced further, the jet may pass over the wall instead of being thrown backward. At some wall angles (including the vertical) this is the only singlejet possibility. In such cases of a forward-going jet, we can also describe the problem as a "weir flow". Vanden-Broeck and Keller [8] computed such weir solutions, but only for small Froude numbers $(F<0.3)$, as their main aim was to compare their numerical results with experimental data. For the supercritical case $(F>1)$, Dias and Tuck [2] solved the problem to determine the range of $F$ where the solutions exist.

Another possible configuration, that can occur if the initial deflection of the stream is nearly vertical, is a free surface with two jets, one forward and one backward. In the case of flow emerging from a nozzle, this type of solution has been computed by Dias and Vanden-Broeck [4] and Vanden-Broeck [7]. Solutions with two sheets covered by one free surface were computed by Dias and Vanden-Broeck [4] for various angles $\beta_{N}$ of the nozzle. A critical value of the Froude number $F_{c}$ which is based on the width of the nozzle is obtained for each value $\beta_{N}$. Therefore, the solutions exist only for $F \geq F_{c}$. When the nozzle is vertical, the flow becomes symmetric. Dias and Vanden-Broeck compared their computations for this case with the results constructed from two pouring flows, by superposing these flows to get the complete free surface of the nozzle flow. This idea was then used to superpose two weir flows to obtain a two-jet solution of the nozzle flow, where each jet was bounded by two free surfaces. The latter solution was then computed by Vanden-Broeck [7] using a finite-difference method for $F \rightarrow \infty$.

For flows in the channel described above, two-jet solutions are computed in the present work. We assume that the flow separates smoothly from the top edge of a 
vertical wall which has a height less than the stagnation value. The fraction $\sigma$ of fluid forming the forward-going jet is one of the quantities that we consider. This parameter $\sigma$ corresponds to a constant scaled value $\psi=\sigma$ of the stream function for the dividing streamline, which splits into two free surfaces at a stagnation point. When $\sigma$ tends to 1 , the limiting case is a forward-jet solution with a stagnation point on the upper free surface. We reproduce this configuration as a special case of the procedure for the two-jet case.

In solving the problem, we use an integral equation formulation as in Wiryanto and Tuck $[9,10]$. Other references for this method can be seen in Goh and Tuck [5] and Tuck [6]. We map the flow domain of the complex potential plane into an artificial $\zeta$-plane. This transformation depends on the fraction $\sigma$ defined above, where $\sigma=\delta / \gamma$ and $\delta, \gamma$ are two parameters corresponding to the images of the stagnation point and of one of the jets in the $\zeta$-plane respectively. We use these two parameters $\delta, \gamma$ as input, and the Froude number $F$ and the scaled height $w$ of the wall are obtained as output. In practice, the latter two parameters would be presented by the physical situation, and our procedure would then inversely determine $\delta, \gamma$, and hence $\sigma$ and the shape of the free surfaces.

\section{Problem formulation}

We consider the two-dimensional flow configuration sketched in Figure 1(a). The flow domain is bounded on the right and below by impermeable plane walls and three free surfaces forming two jets. The fluid is assumed to be inviscid and incompressible and the flow to be steady and irrotational. We choose Cartesian coordinates with the origin at the corner point $\mathrm{C}$; that is, the $x$-axis is chosen to be along the bottom of the channel and the $y$-axis is chosen to be up the vertical wall. Then we write $z=x+\mathrm{i} y$ as the complex coordinate.

For the complex potential $f=\phi+\mathrm{i} \psi$, we introduce the potential function $\phi$ and the stream function $\psi$ as follows. Without loss of generality, we choose $\phi=0$ at the detachment point $\mathrm{D}$, and $\psi=0$ to be the streamline ICDR, the bottom (IC) and vertical (CD) walls having $\phi<0$, and the free surface DR having $\phi>0$. We denote by $Q$ the value of $\psi$ on the incoming free surface streamline IL. The dividing streamline IS and the upper streamline LSR have $\psi=\sigma Q$.

Mathematically, the problem is to find a stream function $\psi(x, y)$ satisfying Laplace's equation $\left(\nabla^{2} \psi=0\right.$ ) within the flow domain, subject to Dirichlet conditions $\psi=$ constant, no flow across the solid boundaries and the free surfaces, and the condition of constant pressure on the free surfaces provided by Bernoulli's equation

$$
\frac{1}{2}\left(\psi_{x}^{2}+\psi_{y}^{2}\right)+g y=\text { constant }
$$




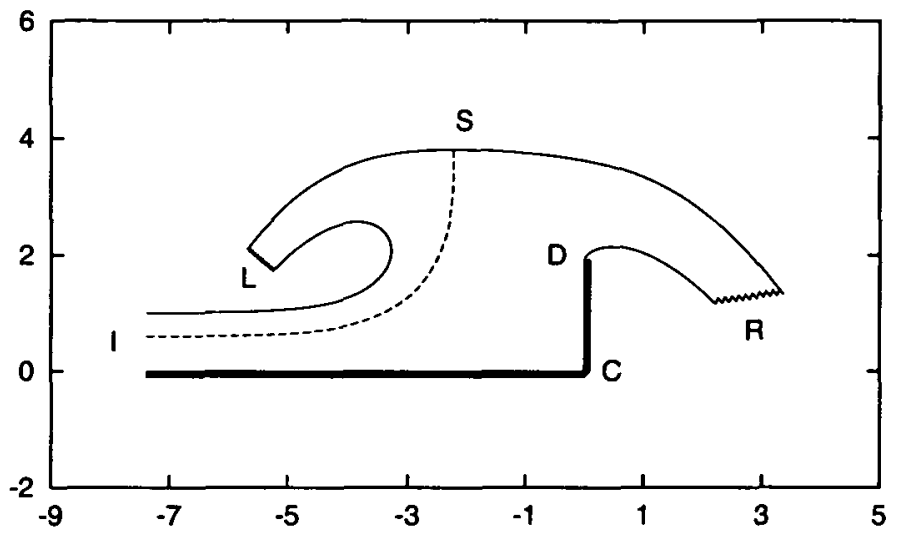

(a)

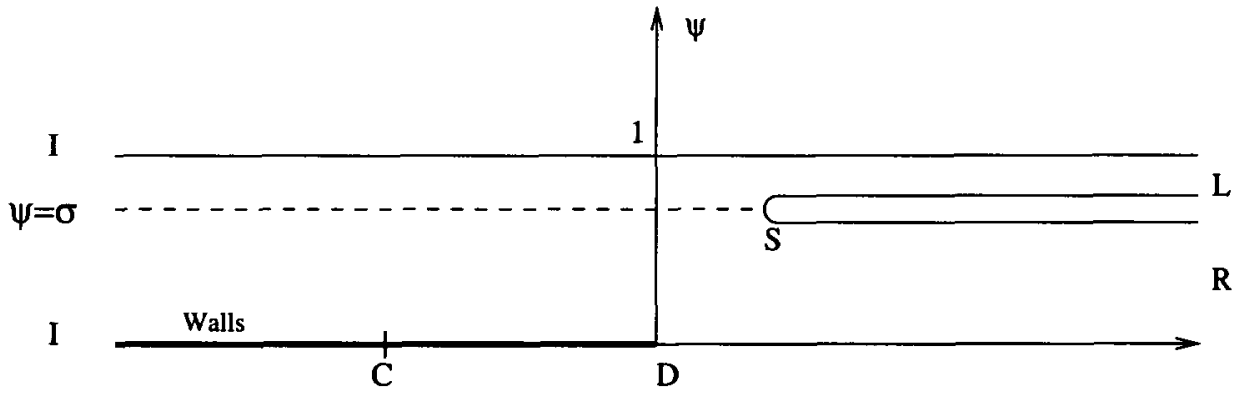

$f$-plane

(b)

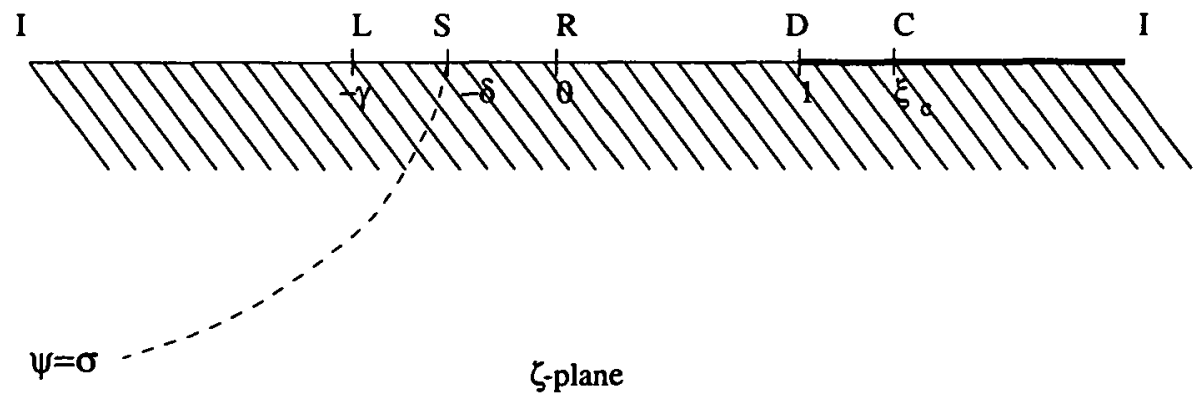

(c)

FigurE 1. (a) Sketch of the flow and the coordinates. (b) The complex potential plane $f=\phi+i \psi$. (c) The complex $\zeta$-plane. 
The problem is non-dimensionalized by taking $U$ as the unit velocity and the depth far upstream $H$ as the unit length. Therefore the volume discharge $Q$ is scaled to unity, and Bernoulli's equation becomes

$$
\frac{1}{2} F^{2}\left(\psi_{x}^{2}+\psi_{y}^{2}\right)+y=\frac{1}{2} F^{2}+1 .
$$

In non-dimensional variables, the flow domain in the $f$-plane becomes an infinite strip of unit height, as shown in Figure 1(b). This is then mapped to a lower half-plane of an artificial variable $\zeta=\xi+$ i $\eta$ shown as the shaded area in Figure 1(c). The streamlines $\psi=0, \psi=1$ and $\psi=\sigma$ corresponding to the upper free surface LSR are mapped to the real axis of $\zeta$ so that the points $\mathrm{L}, \mathrm{R}$ and $\mathrm{D}$ are mapped into the points $-\gamma, 0$ and 1 . The images of the points $\mathrm{C}$ and $\mathrm{S}$ are denoted by $\xi_{c}$ and $-\delta$ respectively. The transformation from the $f$-plane to the $\zeta$-plane can be written as

$$
f=-\frac{1}{\pi}\left[\frac{\delta}{\gamma} \log \zeta+\left(1-\frac{\delta}{\gamma}\right) \log \frac{\zeta+\gamma}{1+\gamma}\right] .
$$

The fraction of fluid $\sigma$ falling beyond the wall can be shown to be equal to $\delta / \gamma$, and the end of the dividing streamline at $\phi=\phi_{s}$ to satisfy

$$
\phi_{s}=-\frac{1}{\pi}\left[\frac{\delta}{\gamma} \log \delta+\left(1-\frac{\delta}{\gamma}\right) \log \left|\frac{\gamma-\delta}{1+\gamma}\right|\right] .
$$

Instead of solving for $\psi$, the problem is inverted to find the complex velocity $d f / d z=u-\mathrm{i} v$ as an analytic function of $\zeta$. To do so, we introduce the hodograph variable

$$
f^{\prime}(z)=e^{\Omega},
$$

where $\Omega(\zeta)=\tau(\zeta)-\mathrm{i} \theta(\zeta)$. The magnitude of the velocity at any point is given by $\left|f^{\prime}(z)\right|=e^{\tau(\zeta)}$ and the angle that any streamline makes with the horizontal is $\theta(\zeta)$. Hence, the kinematic condition on the solid boundaries can be presented in terms of the angle of the wall

$$
\theta(\xi)= \begin{cases}0, & \text { for } \xi_{c}<\xi, \\ \pi / 2, & \text { for } 1<\xi<\xi_{c},\end{cases}
$$

where $\theta(\xi)=\theta(\xi+\mathrm{i} 0)$, and the dynamic condition on the free surfaces becomes

$$
\frac{1}{2} F^{2} e^{2 \tau}+y=\frac{1}{2} F^{2}+1 .
$$

The relation between $\tau$ and $\theta$ can be obtained by applying Cauchy's theorem to the function $\Omega(\zeta) /\left(\zeta-\zeta^{*}\right)$ on a path consisting of the real $\xi$-axis, a semi-circle at 
$|\zeta|=\infty$ in the lower half-plane, and a circle of vanishing radius about the point $\zeta^{*}$. Hence, we have

$$
\Omega\left(\zeta^{*}\right)=-\frac{1}{2 \pi \mathrm{i}} \mathrm{PV} \int_{-\infty}^{\infty} \frac{\Omega(\xi)}{\xi-\zeta^{*}} d \xi
$$

for $\operatorname{Im}\left(\zeta^{*}\right)<0$ since $|\Omega(\zeta)| \rightarrow 0$ as $|\zeta| \rightarrow \infty$. Note that the integral is of Cauchy principal value form, denoted by PV. If we let $\operatorname{Im}\left(\zeta^{*}\right) \rightarrow 0^{-}$, the real part gives

$$
\tau\left(\xi^{*}\right)=\frac{1}{\pi} \mathrm{PV} \int_{-\infty}^{\infty} \frac{\theta(\xi)}{\xi-\xi^{*}} d \xi
$$

where $\xi^{*}=\operatorname{Re}\left(\zeta^{*}\right)$. After substituting the known value of $\theta$ given by (2.5) for $\xi>1$, (2.8) becomes

$$
\tau\left(\xi^{*}\right)=\frac{1}{2} \log \left|\frac{\xi_{c}-\xi^{*}}{1-\xi^{*}}\right|+\frac{1}{\pi} \mathrm{PV} \int_{-\infty}^{1} \frac{\theta(\xi)}{\xi-\xi^{*}} d \xi .
$$

On the other hand, the complex coordinates $z$ and $\zeta$ are related by

$$
\frac{d z}{d \zeta}=-\frac{e^{-\Omega}(\zeta+\delta)}{\pi \zeta(\zeta+\gamma)}
$$

This is obtained from (2.3) and (2.4). For the free surfaces, the major difficulty occurs with integration of (2.10) on the real axis $\zeta=\xi-i 0$, in which the factor $\xi(\xi+\gamma)$ in the dominator makes it essentially impossible to integrate through $\xi=0$ and $\xi=-\gamma$. To avoid these singularities, we evaluate $y$ by integrating (2.10) separately from three different points, namely the top of the wall $\mathrm{D}$ with $y(1)=w$, the upstream point I with $y(-\infty)=1$, and the free-surface stagnation point $S$ with $y(-\delta)=1+\frac{1}{2} F^{2}$. Therefore the $y$-values are

$$
y\left(\xi^{*}\right)= \begin{cases}w-\int_{\xi^{*}}^{1} \frac{d y}{d \xi} d \xi, & \text { for } 0<\xi^{*}<1, \\ 1+\frac{1}{2} F^{2}+\int_{-\delta}^{\xi^{*}} \frac{d y}{d \xi} d \xi, & \text { for }-\gamma<\xi^{*}<0, \\ 1+\int_{-\infty}^{\xi^{*}} \frac{d y}{d \xi} d \xi, & \text { for } \xi^{*}<-\gamma,\end{cases}
$$

with

$$
\frac{d y}{d \xi}=-\frac{e^{-\tau}(\xi+\delta) \sin \theta}{\pi \xi(\xi+\gamma)} .
$$

Equation (2.6) combined with (2.9) and (2.11) is in effect an integral equation for $\theta(\xi)$. Numerically, this equation can be solved by choosing collocation points on the 
free surfaces to construct a system of nonlinear algebraic equations. But this is not yet a closed system. Two other equations must be included. One is the relation between the wall height $w$ and the parameter $\xi_{c}$ obtained by integrating $d y / d \xi$ from 1 to $\xi_{c}$ with $\theta=\pi / 2$, yielding

$$
w=\frac{1}{\pi} \int_{1}^{\xi_{c}} \frac{e^{-\tau}(\xi+\delta)}{\xi(\xi+\gamma)} d \xi
$$

The second equation is obtained by integration of (2.10) along the dividing streamline $\psi(x, y)=\sigma$ for $-\infty<\phi<\phi_{s}$. We only take the imaginary part which is equal to the stagnation level, that is,

$$
\operatorname{Im}\left(-\frac{1}{\pi} \int_{\psi=\sigma} \frac{e^{-\Omega}(\zeta+\delta)}{\zeta(\zeta+\gamma)} d \zeta\right)=\frac{1}{2} F^{2}+1,
$$

where $\Omega$ is computed from (2.7). In order to evaluate the integral in (2.13), we must determine the corresponding curve of the dividing streamline in the $\zeta$-plane. For any point $(\phi, \sigma)$ on this streamline, there is an image point in the $\zeta$-plane which we define as $\zeta=r e^{i \alpha}$ satisfying

$$
\phi=-\frac{1}{\pi}(\sigma \log r+(1-\sigma) \log R)
$$

and

$$
\sigma=-\frac{1}{\pi}(\sigma \alpha+(1-\sigma) \lambda),
$$

where

$$
R=\frac{1}{1+\gamma} \sqrt{r^{2}+\gamma^{2}+2 \gamma r \cos \alpha}
$$

and

$$
\tan \lambda=\frac{r \sin \alpha}{\gamma+r \cos \alpha}
$$

Numerically, (2.14) and (2.15) can be solved by constructing a system of nonlinear equations from $N$ points $\left(\phi_{j}, \sigma\right),-\infty<\phi_{j}<\phi_{s}$ for $j=1,2, \ldots, N$. Then Newton's method is applied to the system to obtain $r_{j}$ and $\alpha_{j}$ corresponding to $\phi_{j}$. This Newton iteration is carried out separately from the computation of the complex integral (2.13). 


\section{Numerical procedure}

Now we describe the numerical procedure to solve the integral equation with the two extra equations (2.12) and (2.13). Our major task is to approximate the integral in (2.9). We truncate the integration domain up to $\xi=-T(T>0)$ representing the free surface far upstream, where $T$ must be chosen large, and we approximate the integration as a summation over $M$ discrete values of $\theta(\xi)$. In order to accomplish this, we need to partition the truncated interval $[-T, 1]$ into $M$ segments. The grid spaces are made unequal so that grid points are crowded near the detachment point $(\xi=1)$, both points representing the jets $(\xi=0$ and $\xi=-\gamma)$, and the stagnation point $(\xi=-\delta)$.

The partition for each subinterval is given as follows. The first subinterval is from $\xi=1$ to $\xi=\epsilon$, where $\epsilon$ is a small input parameter so that the jets are in an interval with $2 \epsilon$-width. The segment end-points are

$$
\xi_{j}=e^{-j^{2} \phi_{1}}, \quad \text { for } j=0,1, \ldots, N,
$$

where $\phi_{1}=-\log \epsilon / N^{2}$. The second subinterval $[-\delta,-\epsilon]$ is divided into $N-1$ segments with end-points

$$
\xi_{2 N+1-j}=-\delta e^{-(j-1)^{3} \phi_{2}}, \quad \text { for } j=1,2, \ldots, N,
$$

where $\phi_{2}=-\log (\epsilon / \delta) /(N-1)^{3}$. Similarly for the third subinterval, from $\xi=-\gamma+\epsilon$ to $\xi=-\delta$, we discretize into segments with end-points

$$
\xi_{2 N+j}=-\gamma+(\gamma-\delta) e^{-(j-1)^{3} \phi_{3}}, \quad \text { for } j=1,2, \ldots, N,
$$

where $\phi_{3}=\log \{(\gamma-\delta) / \epsilon\} / N^{3}$. For the free surface from I to L, we discretize into $2 N$ segments, with end-points

$$
\xi_{3 N+j}=-\gamma-\epsilon e^{(j-1) \phi_{4}}, \quad \text { for } j=1,2, \ldots, 2 N,
$$

where $\phi_{4}=\log \{(T-\gamma) / \epsilon\} /(2 N-1)$. The total number of segments is $M=5 N$.

The Cauchy principal-value integral in (2.9) is approximated by assuming piecewise linear behaviour of the numerator for each segment, giving

$$
\begin{aligned}
\mathrm{PV} \int_{-\infty}^{1} \frac{\theta}{\xi-\xi^{*}} d \xi \approx & \sum_{l=1}^{M}\left(\theta_{l-1}-\theta_{l}\right) \\
& +\left\{\theta_{l}+\left(\theta_{l-1}-\theta_{l}\right) \frac{\xi^{*}-\xi_{l}}{\xi_{l-l}-\xi_{l}}\right\} \log \left|\frac{\xi_{l-1}-\xi^{*}}{\xi_{l}-\xi^{*}}\right|
\end{aligned}
$$


where $\theta_{l}=\theta\left(\xi_{l}\right)$ with $\theta_{0}=\pi / 2$ and

$$
\theta_{2 N}= \begin{cases}0, & \text { for } l=2 N, \\ \pi, & \text { for } l=2 N+1 .\end{cases}
$$

The expression (3.2) represents the streamline $\psi=\sigma$ splitting into two free surfaces with $180^{\circ}$-different directions at the stagnation point $\xi=\xi_{2 N}$.

We replace $\xi^{*}$ in (3.1) by collocation points chosen as the mid-points

$$
\xi_{j}^{*}=\frac{\xi_{j}+\xi_{j-1}}{2}
$$

for $j=1,2, \ldots, N, N+2, \ldots, 3 N, 3 N+2, \ldots, 5 N$, with $\xi_{N+1}^{*}=\epsilon / 2$ and $\xi_{3 N+1}^{*}=$ $-\gamma-\epsilon / 2$. Similarly, $\theta_{j}^{*}=\theta\left(\xi_{j}^{*}\right)$ is defined as the average of $\theta_{j-1}$ and $\theta_{j}$ except that $\theta_{N+1}^{*}$ and $\theta_{3 N+1}^{*}$ are extrapolated linearly from $\theta_{N}$ and $\theta_{N}^{*}$, and from $\theta_{3 N+1}$ and $\theta_{3 N+2}^{*}$ respectively.

The system of nonlinear equations is obtained by substituting the approximate value of (2.9) and (2.11) into (2.6) for each collocation point, and completed with (2.12) and (2.13). This system is used to determine the $M+2$ unknowns, that is, $\theta_{1}, \ldots, \theta_{2 N-1}, \theta_{2 N+1}, \ldots, \theta_{M}, F, w$ and $\xi_{c}$. We choose $\delta$ and $\gamma$ as input quantities, in order to keep fixed the discretization during the iteration process in Newton's method. The IC05NBF package of the NAG routine library was used to solve the system. Most of the computations were performed with $N=40(M=200), \epsilon=0.00005$ and $T=10^{6}$, and with convergence of Newton's method to within error $10^{-8}$. These tolerances are ample to achieve better than 3 significant figure accuracy in all plotted quantities.

\section{Numerical results}

4.1. Two-jet solutions The procedure described in the previous section is used to compute solutions with two jets for various values of $\delta$ and $\gamma$. Once the system of nonlinear algebraic equations is solved, the free-surface profile is evaluated by integrating (2.10) numerically along the $\xi$-axis from $-T$ to 1 . Similarly, integration of (2.10) along $\zeta=\zeta(\phi, \sigma)$ is used to evaluate the coordinate of the dividing streamline $\psi(x, y)=\sigma$ which is terminated at $S$ with $\phi=\phi_{s}$. The $x$-value at the stagnation point is then used to determine the $x$-coordinate of the upper free surface covering the right and left jets.

Typical profiles are shown in Figures 1(a), 2 and 3 for different values of $\delta$ and $\gamma$. A free-surface profile with $\sigma=0.6(\delta=6$ and $\gamma=10)$ is shown in Figure 1(a). The Froude number and the wall height corresponding to this figure are $F=2.370$ and $w=1.929$. The other figures both represent the free-surface profile with $\sigma=0.8$, 


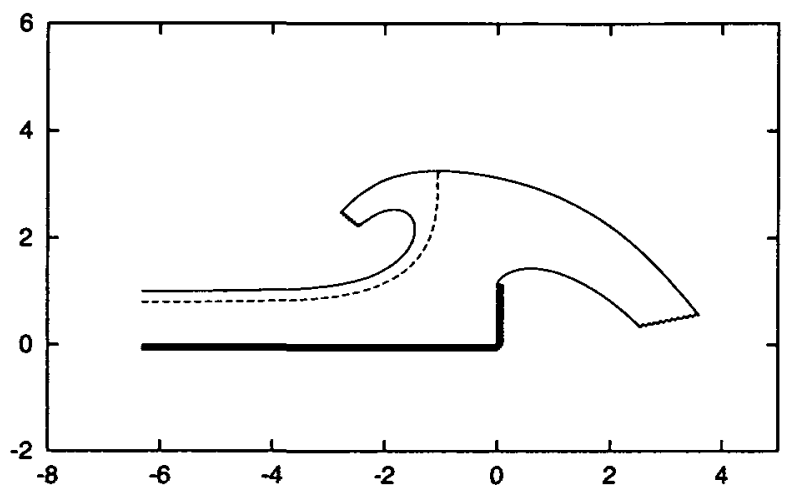

FIGURE 2. A free-surface profile with $\sigma=0.8(\delta=1)$.

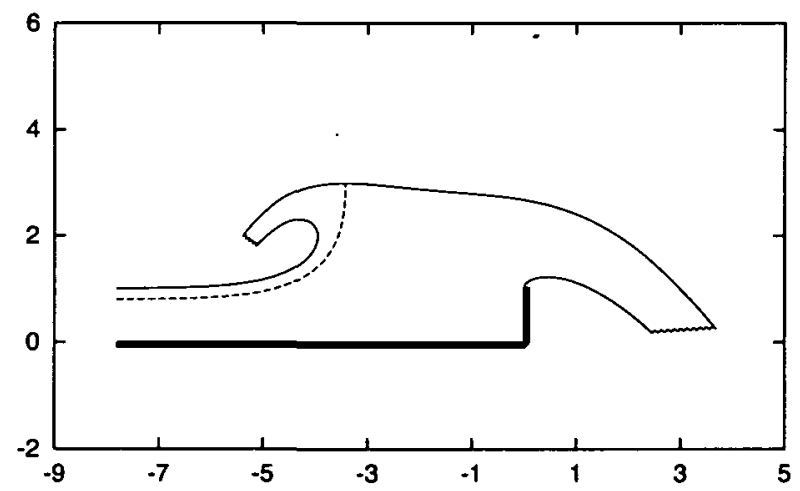

FIGURE 3. Similar to Figure 2 but from $\delta=30$ (still $\sigma=0.8$ ).

but from quite different values $\delta$ and $\gamma$, namely $\delta=1$ corresponding to Figure 2 , and $\delta=30$ for Figure 3. The Froude numbers and the wall heights for these profiles are $F=2.124$ and $w=1.141$ for Figure 2, and $F=1.994$ and $w=1.038$ for Figure 3 .

At a fixed value of $\sigma$ (which is the ratio between $\delta$ and $\gamma$ ), our computations show that the Froude number decreases on increasing $\delta$. This is accompanied by decreasing the wall height until a certain level $w_{\min }$ is reached, and then the wall height increases again. A plot of both physical quantities $w$ and $F$ is shown in Figure 4 for three different values of $\sigma$, namely $0.6,0.7$ and 0.8 . The last curve is for $\sigma=1$, to be discussed below.

Along the curves $\sigma=$ constant, the splash seems to move farther and farther upstream from the wall as we decrease the Froude number. This shifting is shown by an increase in the horizontal distance $d$ between the stagnation point and the vertical wall. As an illustration, we can see the free-surface profile in Figure $2(F=2.124)$ and Figure $3(F=1.994)$ with $d=1.061$ and 3.428 respectively. Other values of $d$ 


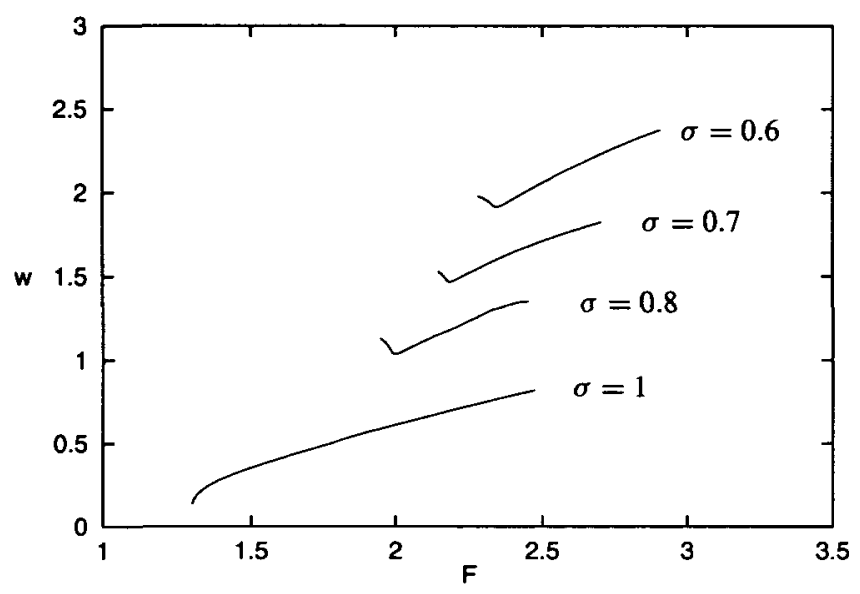

FIGURE 4. Plots of relative wall height $w=W / H$ versus Froude number $F$, for various values of the flux fraction $\sigma$ in the forward-going jet.

are shown in Figure 5 plotted versus the Froude number $F$, for the three values of $\sigma$ given above.

Our results suggest that, at each fixed value of $\sigma$, there is a minimum Froude number $F_{c}(\sigma)$. For example, Figure 5 suggests that $d \rightarrow \infty$ as $F \rightarrow F_{c}$ from above. Our plots of free surfaces suggest that, for large values of $d$, a uniform stream is formed downstream before the wall is reached. Therefore, this configuration may be considered as a generalisation of the problem solved by Dias and Tuck [3]. The flow is then from left to right in a channel without a wall, such that the stream is uniform on both sides of the jet, supercritical far upstream and subcritical far downstream. Although there is now only a single backward-going jet, the parameter $\sigma$ still measures the fraction of the stream that continues to the right. If we let $\sigma \rightarrow 0$, we should then recover the unique solution of Dias and Tuck [3] with $F_{c}(0)=2.994$. Although we have not computed results for small enough $\sigma$ to approach this limit, the trend in Figure 5 is consistent with it.

4.2. Solutions with a stagnation point In the formulation in the previous section, the parameter $\delta$ can be allowed to be either 0 or $\gamma$, with consequent disappearance of one of the two jets. For the first case $\delta=0$, the formulation represents a flow with a backward-going jet only. The end of the dividing streamline $S$ then corresponds to

$$
\phi=-\frac{1}{\pi} \log \frac{\gamma}{\gamma+1}
$$

on $\psi=0$ which is mapped to $\zeta=0$. But from our knowledge of the exact zerogravity solutions (see [2]), we expect that such solutions exist only for wall inclination 


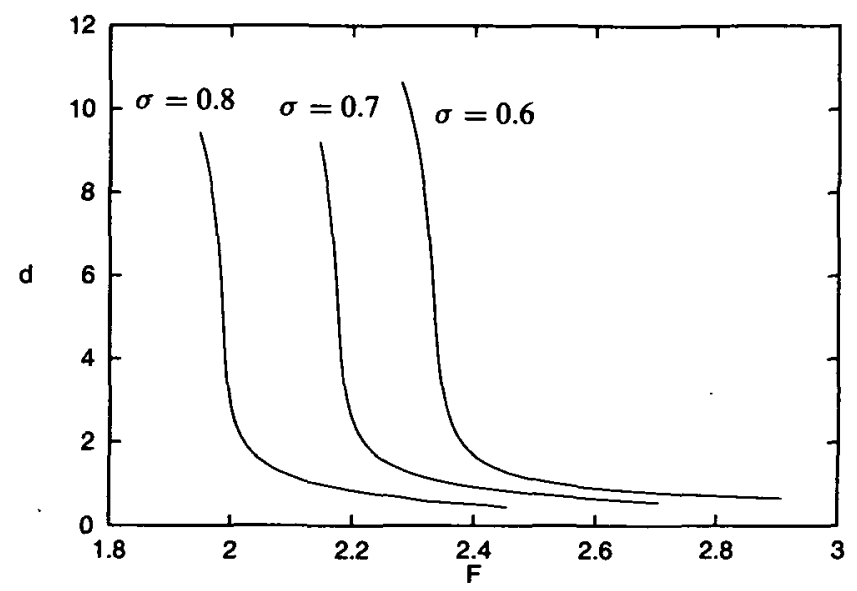

FIGURE 5. Plots of distance $d$ between stagnation point and wall versus $F$, for various $\sigma$.

$\beta>\pi / 2$. Therefore, we do not consider this case here, since we have $\beta=\pi / 2$ in the present paper.

The second case $\delta=\gamma$ (or $\sigma=1$ ) represents a flow with a forward-going jet only. Our transformation is then

$$
f(\zeta)=-\frac{1}{\pi} \log \zeta
$$

which is obtained by taking $\delta \rightarrow \gamma$ in (2.3). Therefore, the stagnation point is at $\phi=-\log \gamma / \pi$ on $\psi=1$. This point is mapped to $\zeta=-\gamma$ by (4.1).

On the other hand, the transformation (4.1) gives a relation between the complex coordinate $z$ and $\zeta$

$$
\frac{d z}{d \zeta}=-\frac{e^{-\Omega}}{\pi \zeta}
$$

so that the $y$-values of the free surfaces are evaluated from

$$
y\left(\xi^{*}\right)= \begin{cases}w-\int_{\xi^{*}}^{1} \frac{d y}{d \xi} d \xi, & \text { for } 0<\xi^{*}<1 \\ \frac{1}{2} F^{2}+1+\int_{-\gamma}^{\xi^{*}} \frac{d y}{d \xi} d \xi, & \text { for }-\gamma<\xi^{*}<0 \\ \frac{1}{2} F^{2}+1-\int_{\xi^{*}}^{-\gamma} \frac{d y}{d \xi} d \xi, & \text { for } \xi^{*}<-\gamma\end{cases}
$$

where $d y / d \xi=-e^{-\tau} \sin \theta /(\pi \xi)$. For the upper free surface, we integrate (4.2) starting from the stagnation point so that it is possible to reach non-unit depth as 


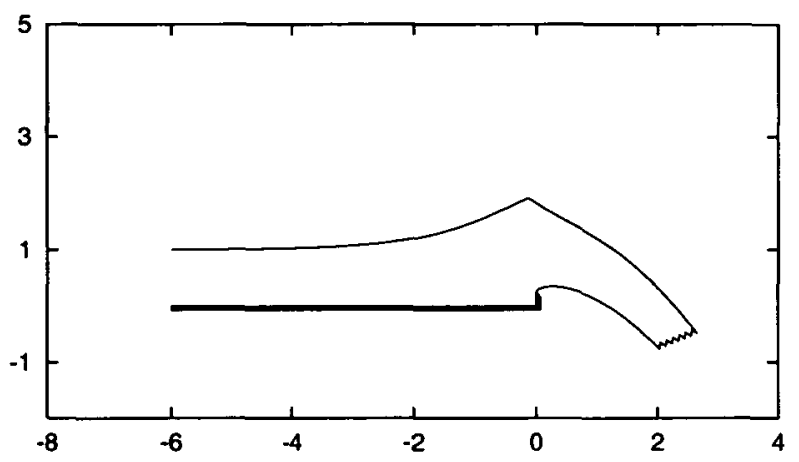

FIGURE 6. A free-surface profile with a $120^{\circ}$ stagnation point on the upper free surface, obtained in the limit when $\sigma=1$. The wall height is $w=0.243$.

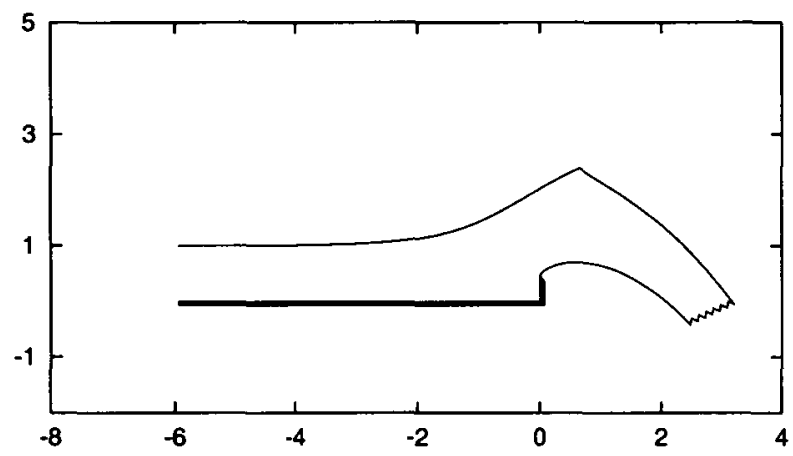

FIGURE 7. Similar to Figure 6, with $w=0.450$.

$\xi^{*} \rightarrow-T$. To avoid this, we use a boundary condition

$$
\frac{1}{2} F^{2}-\int_{-T}^{-\gamma} \frac{d y}{d \xi} d \xi=0
$$

which contributes in constructing the system of nonlinear equations.

Following the procedure in the previous section, $M+2$ equations are obtained, that is, $M$ equations from the collocation points, equation (2.12) by substituting $\delta=\gamma$ and equation (4.4). These are used to solve for $M+3$ unknowns $\theta_{j},(j=1,2, \ldots, M)$, $F, w$ and $\xi_{c}$. The $(M+3)$-rd equation is obtained by imposing the constraint that the upper free surface makes a 120-degree angle at the stagnation point. If $\theta_{2 N}$ is the angle at the stagnation point and $\theta_{2 N}^{-}$is the angle at the same point extrapolated from $\theta_{2 N+1}$ and $\theta_{2 N+2}$, we have

$$
\theta_{2 N}^{-}-\theta_{2 N}=\frac{\pi}{3}
$$




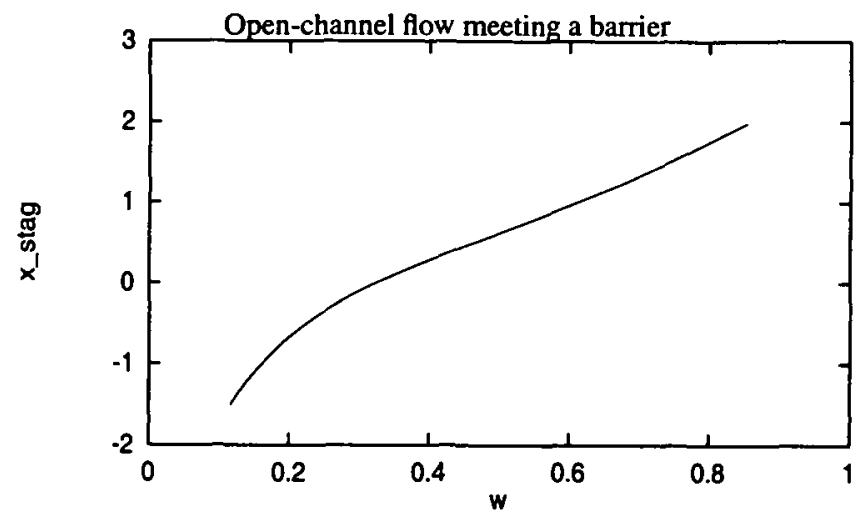

FIGURE 8. Variation with wall height $w$ of the $x$-coordinate $x_{\text {stag }}$ of the free-surface stagnation point, for solutions as in Figures 6 and 7 with $\sigma=1$.

Here we use subtraction since the angle of the free surface for $-\gamma<\xi<0$ must be negative. Then the system is solved by Newton's method.

Two free-surface profiles are shown in Figures 6 and 7 for $\gamma=1$ and 0.1 respectively. The corresponding physical quantities are $F=1.354$ and $w=0.243$ for Figure 6, and $F=1.668$ and $w=0.450$ for Figure 7 . It is interesting to note from these profiles that the stagnation point moves forward as the wall height is increased. We confirm this by plotting the $x$-coordinate of the stagnation point $x_{\text {stag }}$ versus $w$ in Figure 8. This shifting of the stagnation point agrees with the results in Dias and Tuck [2] for the case of a free-surface flow without a stagnation point.

The plot of $F$ versus $w$ for solutions with a stagnation point is shown together with the previous results as the curve $\sigma=1$ in Figure 4 . In contrast with the other curves for $\sigma<1$, we found that this case gives a monotone relationship between $w$ and $F$.

\section{Conclusions}

We have performed accurate numerical calculations for a free-surface flow consisting of a stream with incoming speed $U$, splitting into two jets in a channel of finite depth $H$ blocked by a vertical wall of height $W$. We obtained a relationship between $F=U / \sqrt{g} \bar{H}$ and $w=W / H$ for fixed values of the fraction $\sigma$ of fluid forming the forward-going jet. If we increase this parameter $\sigma$ toward 1 , the relationship tends to the boundary of a region where solutions with a forward-going jet exist. On the other hand, our procedure is not able to obtain any two-jet solutions for $\sigma<0.4$. Nevertheless, two-jet solutions exist with a wall height which is less than the stagnation height at a fixed Froude number. We can conclude this from the limiting case $\sigma \rightarrow 0$, which gives $\phi_{s}>0$ on the streamline $\psi=0$.

This present work contributes in obtaining transition solutions between forward- 
jet solutions as in Dias and Tuck [2], and backward-jet solutions with a stagnation point on the wall as in Dias and Christodoulides [1] and Wiryanto and Tuck [9]. Unfortunately, the two-jet configurations computed here still do not cover the whole gap in the parameter range. Further work is needed on this matter. Determination of the limiting solution at the minimum Froude number $F=F_{c}(\sigma)$ is another interesting problem, that could be examined as a generalisation of the steady breaking wave of Dias and Tuck [3].

\section{Acknowledgements}

The first author is a staff member of the Department of Mathematics, Bandung Institute of Technology, Ganesha Street 10 Bandung, Indonesia. The present work was carried out when he was a PhD student at the University of Adelaide, supported by Ausaid.

\section{References}

[1] F. Dias and P. Christodoulides, "Ideal jets falling under gravity", Phys. Fluids A 3 (1991) 17111717.

[2] F. Dias and E. O. Tuck, "Weir flows and waterfalls", J. Fluid Mech. 230 (1991) 525-539.

[3] F. Dias and E. O. Tuck, “A steady breaking wave”, Phys. Fluids A 5 (1993) 277-279.

[4] F. Dias and J.-M. Vanden-Broeck, "Flows emerging from a nozzle and falling under gravity", $J$. Fluid Mech. 213 (1990) 465-477.

[5] M. K. Goh and E. O. Tuck, "Thick waterfalls from horizontal slots", J. Engng. Maths. 19 (1985) 341-349.

[6] E. O. Tuck, "Efflux from a slit in a vertical wall", J. Fluid Mech. 176 (1987) 253-264.

[7] J.-M. Vanden-Broeck, "Two-dimensional jets aimed vertically upwards", J. Austral. Math. Soc. Ser. B 34 (1993) 393-400.

[8] J.-M. Vanden-Broeck and J. Keller, "Weir flows", J. Fluid Mech. 176 (1987) 283-293.

[9] L. H. Wiryanto and E. O. Tuck, "A back-turning jet formed by a uniform shallow stream hitting a vertical wall", in Intermational Conference on Differential Equations, Inst. Tek. Bandung, Indonesia, October 1996 (eds. B. van Groesen and E. Soewono), (Kluwer, 1997) 371-379.

[10] L. H. Wiryanto and E. O. Tuck, "A boundary-element solution of a free-surface flow in a blocked channel", in 8th Computational Techniques and Applications Conference, Adelaide, September 1997 (eds. J. Noye, M. Teubner and A. Gill), (World Scientific, Singapore, 1998) 743-750. 